\title{
Incidência de malformações congênitas em crianças concebidas através de injeção intracitoplasmática de espermatozóides
}

\author{
Incidence of congenital malformations in children conceived through intracytoplasmic \\ sperm injection
}

Edilberto de Araújo Filho ${ }^{1}$, Sonia Ventura Carillo², Patricia Gomes Silva ${ }^{3}$, Ciro Dresch Martinhago ${ }^{4}$, Ricardo Luiz Razera Baruffi ${ }^{5}$, João Batista Alcantara Oliveira ${ }^{6}$, José Gonçalves Franco $\mathrm{Jr}^{7}$

\section{RESUMO}

Objetivo: avaliar a incidência e tipos de malformações congênitas maiores (MCM) em crianças concebidas por injeção intracitoplasmática de espermatozóides (ICSI) e nascidas vivas. Métodos: um total de 680 crianças nasceram vivas de 511 casais submetidos à ICSI no período de janeiro de 1999 a dezembro de 2002. A coleta de dados das crianças foi procedida por meio de questionário padronizado e exame clínico. Dos 511 casais, 366 foram contatados para amostragem de 371 gestações. Das 680 crianças nascidas vivas, 520 foram avaliadas, 250 delas (48,1\%) por meio de questionário e 270 (51,9\%) por questionário e exame físico. Duzentas e cinqüenta crianças foram de gestação única e 270 de gestação múltipla. Na análise das malformações congênitas foi empregada a $10^{\mathrm{a}}$ Revisão da Classificação Internacional de Doenças. Nesse estudo foram analisadas apenas as MCM. A incidência de MCM foi comparada à da população geral obtida pelo Estudo Colaborativo Latino-Americano de Malformações Congênitas. A análise estatística foi feita usando o teste do $\chi^{2}$ (nível de significância p<0,05). Resultados: das 520 crianças observadas, 15 apresentaram MCM, dando incidência de 2,9\%. Não houve diferença significativa em relação ao grupo controle ( $p>0,05)$, que teve $2,6 \%$ de incidência de MCM. As malformações mais freqüentes foram as de origem cardíaca (quatro isoladas e duas associadas), correspondendo a 40\% do total. Os outros tipos de MCM foram: renal (três), defeito de fechamento do tubo neural (dois), defeito do crânio (um), lábio leporino (um), genital (um), síndrome de Down (associada à cardiopatia) (dois) e músculo-esquelética (um). Seis MCM ocorreram em crianças provenientes de gestações únicas e nove de gestações múltiplas. Conclusão: as crianças concebidas por ICSI e nascidas vivas apresentaram incidência de malformações congênitas maiores $(2,9 \%)$ próximo ao esperado para a população geral (2,6\%). Entretanto, para estabelecer com precisão os riscos de MCM é necessária continuidade na avaliação das crianças concebidas por ICSI.

PALAVRAS-CHAVE: Técnicas reprodutivas assistidas; Injeções de esperma intracitoplasmáticas; Anormalidades

\section{ABSTRACT}

Purpose: to evaluate the incidence and types of major congenital malformations (MCM) in liveborn children conceived by intracytoplasmic sperm injection(ICSI). Methods: a total of 680 liveborn children resulted from 511 couples submitted to ICSI from January, 1999 to December, 2002. Data collection of the children was performed through standardized questionnaire and clinical examination. Of the 511 couples, 366 had been contacted for a sampling of 371 gestations. Of the 680 liveborn, 520 had been evaluated, 250 of them (48.1\%) through questionnaire and $270(51.9 \%)$ through questionnaire and physical examination. Two hundred and fifty children were from singleton pregnancies and 270 from multiple pregnancies. Malformations were

Trabalho realizado no Centro de Reprodução Humana Prof Franco Jr - Ribeirão Preto (SP), Brasil.

1 Pós-Graduando do Programa de Pós-Graduação em Ginecologia, Obstetrícia e Mastologia - Departamento de Ginecologia e Obstetrícia - Faculdade de Medicina de Botucatu - Universidade Estadual Paulista Julio de Mesquita - UNESP - Botucatu (SP), Brasil.

2 Médica Pediatra do NADI - Núcleo de Acompanhamento do Desenvolvimento Infantil - Ribeirão Preto (SP), Brasil.

3 Psicóloga do NADI - Núcleo de Acompanhamento do Desenvolvimento Infantil - Ribeirão Preto (SP), Brasil.

4 Médico Geneticista do NADI - Núcleo de Acompanhamento do Desenvolvimento Infantil - Ribeirão Preto (SP), Brasil.

5 Médico Ginecologista do Centro de Reprodução Humana Prof Franco Jr / NADI - Núcleo de Acompanhamento do Desenvolvimento Infantil - Ribeirão Preto (SP), Brasil.

6 Médico Ginecologista do Centro de Reprodução Humana Prof Franco Jr / NADI - Núcleo de Acompanhamento do Desenvolvimento Infantil - Ribeirão Preto (SP), Brasil.

7 Diretor do Centro de Reprodução Humana Prof Franco Jr / Diretor do NADI - Núcleo de Acompanhamento do Desenvolvimento Infantil / Professor do Programa de Pós-Graduação em Ginecologia, Obstetrícia e Mastologia - Departamento de Ginecologia e Obstetrícia - Faculdade de Medicina de Botucatu - Universidade Estadual Paulista Julio de Mesquita - UNESP - Botucatu (SP), Brasil.

Correspondência: José Gonçalves Franco Jr

Centro de Reprodução Humana Prof Franco Junior

Av. Prof. João Fiusa, 689 - 14025-310 - Ribeirão Preto - SP - Tel/Fax: (16) 3911-1100 - e-mail: crh@crh.com.br ou franco@crh.com.br 
classified according to the 10th revision of the International Statistical Classification of Diseases and Related Health. Only MCM were analyzed in this study. The incidence of MCM was compared with that of the general population obtained by the Latin American Collaborative Study of Congenital Malformations. The statistical analysis was performed by the $\chi^{2}$ test (level of significance $\mathrm{p}<0.05$ ). Results: of the 520 children, 15 presented MCM, resulting in an incidence of $2.9 \%$. There was no difference in relation to the control group ( $\mathrm{p}>0.05$ ), which showed $2.6 \%$ incidence of MCM. The most frequent malformations were of cardiac origin (four isolated and two associated), corresponding to $40 \%$ of the total. The other types of MCM were: renal (three), neural tube (two), skull (one), cleft lip (one), genital (one), Down syndrome (associated with cardiac malformations) (two), and musculoskeletal (one). Six MCM occurred in children from singleton pregnancies and nine in children from multiple pregnancies. Conclusion: the liveborn children conceived by ICSI presented incidence of major congenital malformations $(2.9 \%)$ near to the expected for the general population (2.6\%). However, to establish the risks of MCM with precision it is necessary to continue the evaluation of the children conceived by ICSI.

KEYWORDS: Reprotuctive techniques, assisted; Sperm injections, intracytoplasmic; Abnormalities

\section{Introdução}

Com a evolução do conhecimento, aumentou o interesse da medicina pelas malformações congênitas observadas nos seres humanos, assim como a procura por explicações para tais eventos. Neste contexto, as técnicas de reprodução assistida (TRA) são vistas como mais um ponto de questionamento. Se por um lado, nesses mais de 27 anos de seu desenvolvimento, tornaram-se ferramentas indispensáveis no tratamento do casal infértil, revolucionando o arsenal terapêutico, por outro, persistem preocupações quanto às repercussões sobre as crianças concebidas com a aplicação dessas técnicas ${ }^{1-8}$. A possibilidade de aumento da incidência de malformações congênitas é uma das grandes discussões, objeto de vários estudos que realçam a necessidade de contínuo monitoramento das crianças concebidas por TRA.

O surgimento e a rápida difusão da injeção intracitoplasmática de espermatozóides (ICSI) ampliaram as preocupações quanto à segurança das crianças resultantes das TRA. Desde o princípio a ICSI foi considerada uma técnica de risco pelos pesquisadores, por ser mais invasiva do que a fertilização in vitro (FIV) tradicional. As preocupações quanto à segurança da ICSI estão relacionadas à técnica em si, à constituição genética do espermatozóide usado, além da possibilidade de imprinting genômico incompleto no momento da fertilização devido à utilização de espermatozóides testiculares ${ }^{5,6,9,10}$.

Trabalhos publicados não indicam aumento na incidência de malformações congênitas nas crianças nascidas de ICSI em comparação com a população geral ${ }^{6,11,12}$. No entanto, diferentes estudos referem aumento de alterações cromossômicas, especialmente as chamadas de novo: 3,3\% (7/209) de anormalidades cromossômicas, das quais seis de novo e uma herdada ${ }^{11} 2,9 \%$ (42/1437) de cariótipos anormais, dos quais 23 de novo e 19 herdados (15 herdados do pai) ${ }^{12} ; 1,5 \%$ (17/1136) de cariótipos anormais, dos quais 14 de novo e 3 herdados ( 2 herdados do pai) ${ }^{13}$. Estima-se em aproximadamente $5 \%$ o risco de anormalidades cromossômicas para crianças de $\operatorname{ICSI}^{12}$ devido às aberrações cromossômicas nos genitores, mais freqüentemente pais ${ }^{14}$, porcentagem muito mais elevada do que o valor de $0,5 \%$ previsto para a população geral ${ }^{15}$.

Por outro lado, trabalhos recentes e envolvendo amostras populacionais maiores mostraram taxas de malformações congênitas superiores aos controles ${ }^{2-4,16,17}$. Entretanto, enquanto algumas publicações não diferenciaram FIV de ICSI em sua análise, outras tiveram "bias" principalmente quanto ao recrutamento da amostra populacional de controle. Assim, ainda persistem questionamentos quanto à incidência de malformações congênitas nas crianças concebidas por ICSI.

O acompanhamento das crianças na América Latina é difícil, pois os centros de reprodução humana que executam TRA não dispõem de estruturas organizadas para este fim. A Rede Latino-americana de Reprodução Assistida (REDLARA) tem um registro anual de seus resultados, inclusive de malformações congênitas após TRA ${ }^{18}$. Contudo, são reportados dados sobre malformação congênita de apenas $30 \%$ das crianças nascidas, com a maioria dos centros credenciados da REDLARA referindo perda de seguimento como justificativa para falha no envio das informações. No Brasil não há um registro específico sobre as crianças advindas de TRA.

Entendendo a importância de dados que reflitam a nossa realidade, este estudo procura analisar a incidência de malformações congênitas em crianças brasileiras provenientes de ICSI e dessa forma obter informações consistentes que ajudem a entender as conseqüências e limites de segurança do uso dessa técnica. 
Métodos

Esse estudo foi conduzido no Centro de Reprodução Humana (CRH) Prof. Franco Junior após aprovação pelo Comitê de Ética e Pesquisa da Universidade de Ribeirão Preto (UNAERP). Foi realizada análise populacional retrospectiva observando a taxa de malformações congênitas maiores (MCM) em crianças concebidas por meio da utilização da ICSI realizada no período de janeiro de 1999 a dezembro de 2002 e nascidas vivas.

Foi incluído inicialmente um total de 644 casais cujas parceiras engravidaram após ICSI, e das quais 115 abortaram. Quinhentas e vinte e nove mulheres levaram a gestação além da $24^{a}$ semana e entre gestações únicas e múltiplas nasceram 698 crianças, 18 destas natimortas. Portanto, 680 crianças nasceram vivas de 511 casais, sendo essa a população alvo do estudo.

Os critérios de inclusão considerados foram: crianças concebidas pela aplicação da ICSI executada no período estipulado e nascidas vivas. Foram consideradas as crianças nascidas após descongelamento de embriões excedentes e aquelas geradas pelo programa de doação de óvulos desde que a ICSI tivesse sido empregada. Os espermatozóides utilizados na ICSI foram provenientes de ejaculação ou colhidos por punção do epidídimo ou biópsia do testículo. Em 39 casos foram utilizados espermatozóides previamente congelados por razões diversas (casos de azoospermia em que não se encontraram espermatozóides à biópsia e/ou punção e impossibilidade de comparecimento no dia da coleta dos óvulos).

Os critérios de exclusão foram: casos de coito programado, inseminações intra-uterinas, as FIV clássicas, FIV associado à ICSI ou ICSI associada a biópsia embrionária para diagnóstico genético pré-implantacional.

Rotineiramente, o ácido fólico na dose de 5 $\mathrm{mg} /$ dia foi prescrito para as pacientes, tendo início à época da transferência embrionária e mantido até a $12^{\text {a }}$ semana de gestação.

Para a coleta dos dados, foi elaborado questionário pela equipe do Núcleo de Atendimento e Desenvolvimento Infantil (NADI). Esse questionário é dividido em três partes. A primeira é direcionada às informações sobre os pais individualmente, buscando dados que possam ter repercussão sobre a prole, além de caracterizar a população: idades materna e paterna na época do parto, hábitos pessoais (fumo, uso de bebidas alcoólicas, drogas ou medicamentos), profissão, doenças pessoais e familiares (diabete, hipertensão, doenças genéticas). Nesta parte também estão presentes questões que fornecem informações sociais sobre a família, como renda, existência de outros filhos (naturais, adotados ou também provenientes de reprodução assistida). Na segunda parte as perguntas são direcionadas a evolução da gravidez e às condições de nascimento: duração da gestação, uso de medicações, intercorrências (sangramentos, amniorrexe, etc.), data do parto, tipo de parto, peso e estatura, índice de Apgar, intercorrências ao nascimento (necessidade de UTI neonatal, uso de respirador, depressão respiratória, etc.). Há também o questionamento sobre a presença ou não de malformações. A terceira parte objetiva informações sobre a saúde da criança (alimentação, doenças intercorrentes, desenvolvimento motor) e também sobre a evolução das malformações existentes (correção cirúrgica, uso crônico de medicações). Além disso, há informações sobre malformações que tenham passado desapercebidas ao nascimento ou se manifestado mais tardiamente.

Os casais foram contatados via correio ou correio eletrônico, sendo orientados sobre o trabalho e convidados a visitar o NADI para que seus filhos fossem examinados. Junto ao convite enviávamos o questionário para preenchimento caso o comparecimento fosse impossivel. $\mathrm{Na}$ entrevista, o questionário era preenchido e as crianças eram examinadas pela equipe multidisciplinar. Os casais que não compareciam ou não enviavam o questionário eram contatados via telefone, pelo que se entrevistava um dos pais da(s) criança(s), empregando as perguntas constantes no questionário padrão.

Dos 511 casais, $366(71,6 \%)$ foram contatados para amostragem de 371 gestações (cinco casais tiveram duas gestações no período do estudo). A perda de seguimento dos casais foi $28,4 \%$ (145/ 511 ). As características dos pais estão resumidas na Tabela 1. A média de idade das mães na época do parto foi de $34 \pm 4,9$ anos e a dos pais $37,7 \pm 6,9$ anos. Duzentas e setenta e três mães $(74,6 \%)$ e 268 pais $(73,2 \%)$ têm nivel superior de escolaridade. Um total de 359 pais $(98,1 \%)$ e 277 mães $(75,7 \%)$ são profissionalmente ativos. Analisando a renda familiar, foi observado que 215 casais têm renda superior a $R \$ 5.000,00$ mensais $(58,8 \%)$, sendo 92 deles com renda superior a $\mathrm{R} \$ 10.000,00(24,9 \%)$. Noventa e três mães $(25,4 \%)$ e 116 pais $(31,7 \%)$ referiram algum tipo de problema de saúde, que variava de simples alergia até doenças mais sérias como obesidade, hipertensão e diabete. Algum grau de depressão e ansiedade foi relatado por 25 mulheres $(6,9 \%)$ e por apenas sete homens $(1,9 \%)$. Dentre as mulheres, $22(6 \%)$ eram fumantes, mas somente três $(0,8 \%)$ confirmaram o uso durante a gestação. Com relação ao uso de álcool, somente $12(3,3 \%)$ mães fizeram algum tipo de ingestão de álcool durante a gestação. 
Tabela 1 - Características familiares: resumo dos aspectos médicos e socioeconômicos dos 366 casais incluídos no estudo.

\begin{tabular}{|c|c|c|c|c|c|c|}
\hline \multirow{2}{*}{ Idade(ao nascimento) } & \multicolumn{3}{|c|}{$\begin{array}{c}\text { Mãe } \\
\mathrm{n}=366\end{array}$} & \multicolumn{3}{|c|}{$\begin{array}{c}\text { Pai } \\
\mathrm{n}=366\end{array}$} \\
\hline & & & & & & \\
\hline \multirow[t]{4}{*}{ Escolaridade } & Fundamental & $3,8 \%$ & $(14 / 366)$ & Fundamental & $6,0 \%$ & $(22 / 366)$ \\
\hline & Médio & $21,6 \%$ & $(79 / 366)$ & Médio & $20,8 \%$ & $(76 / 366)$ \\
\hline & Superior & $59,8 \%$ & $(219 / 366)$ & Superior & $58,7 \%$ & $(215 / 366)$ \\
\hline & Pós-graduação & $14,8 \%$ & $(54 / 366)$ & Pós-graduação & $14,5 \%$ & $(53 / 366)$ \\
\hline \multirow[t]{2}{*}{ Atividade profissional } & Ativa & $75,7 \%$ & $(277 / 366)$ & Ativo & $98,1 \%$ & $(359 / 366)$ \\
\hline & Inativa & $24,3 \%$ & $(89 / 366)$ & Inativo & $1,9 \%$ & $(07 / 366)$ \\
\hline \multirow[t]{4}{*}{ Renda mensal familiar } & & & $\$ \$ 1.000,00$ & & $2,7 \%$ & $(10 / 366)$ \\
\hline & & & $0-5.000,00$ & & $38,5 \%$ & $(141 / 366)$ \\
\hline & & & $-10.000,00$ & & $33,9 \%$ & $(124 / 366)$ \\
\hline & & & $\$ 10.000,00$ & & $24,9 \%$ & $(91 / 366)$ \\
\hline \multirow[t]{3}{*}{ Problemas de saúde } & Negam & $74.6 \%$ & $(273 / 366)$ & Negam & $68,3 \%$ & $(250 / 366)$ \\
\hline & Referem & $25.4 \%$ & $(93 / 366)$ & Referem & $31,7 \%$ & $(116 / 366)$ \\
\hline & Obs: depressão/ansiedade & $6,9 \%$ & $(25 / 366)$ & Obs: depressão/ansiedade & $1,9 \%$ & $(7 / 366)$ \\
\hline \multirow[t]{2}{*}{ Fumo } & Não & $94 \%$ & $(344 / 366)$ & Não & $87.4 \%$ & $(320 / 366)$ \\
\hline & Sim & $6 \%$ & $(22 / 366)$ & Sim & $12.6 \%$ & $(46 / 366)$ \\
\hline \multirow[t]{3}{*}{ Álcool } & Não & $58,5 \%$ & $(214 / 366)$ & Não & $29 \%$ & $(106 / 366)$ \\
\hline & Sim & $41,5 \%$ & $(152 / 366)$ & Sim & $71 \%$ & $(260 / 366)$ \\
\hline & Na gestação & $3,3 \%$ & $(12 / 366)$ & & & \\
\hline Outras drogas & \multicolumn{3}{|c|}{$100 \%$ das pacientes negam uso } & \multicolumn{3}{|c|}{$100 \%$ dos pacientes negam uso } \\
\hline
\end{tabular}

Das 680 crianças nascidas vivas, 520 foram avaliadas, com uma taxa de perda de seguimento de $23,5 \%(160 / 680)$. As falhas ocorreram por impossibilidade de contato, dificuldades provenientes da distância ou recusa dos pais em fornecer informações e/ou submeter os filhos a exame. O questionário foi aplicado a 250 delas $(48,1 \%)$ e questionário e exame físico a 270 (51,9\%).

A média de idade dessas crianças no momento da avaliação foi de 3 anos e 5 meses \pm 13 meses (variando de 1 a 5 anos). Do total das 520 crianças avaliadas, $276(53,1 \%)$ eram do sexo masculino e $244(46,9 \%)$ do feminino. A distribuição regional mostrou que $412(79,2 \%)$ moram na região Sudeste, principalmente no estado de São Paulo, $58(11,2 \%)$ na região Nordeste, 21 (4,0\%) na região Sul, $18(3,5 \%)$ na região Centro-Oeste e 7 $(1,3 \%)$ na região Norte. Quatro crianças $(0,8 \%)$ estavam morando fora do Brasil na época da avaliação.

Duzentas e cinqüenta crianças foram de gestação única $(48,1 \%), 194$ de gemelar $(37,3 \%), 60$ trigemelar $(11,5 \%)$ e 16 quadrigemelar $(3,1 \%)$. A duração média das gestações foi de $36 \pm 2,7$ semanas, sendo que nas únicas foi de $37,8 \pm 2.2$ semanas, nas gemelares $35,3 \pm 2,3$ semanas, nas trigemelares $33,5 \pm 1,8$ semanas e quadrigemelares $31,0 \pm 1,5$ semanas.

Quanto aos procedimentos laboratoriais, em $32,1 \%(167 / 520)$ das crianças avaliadas algum procedimento adicional à ICSI foi realizado: descongelamento de embrião: 94 crianças $(18,1 \%)$; doação de óvulos: 34 (6,5\%); utilização de espermatozóides provenientes de aspirados do epidídimo (PESA: percutaneous epididymal sperm aspiration) ou obtidos por biópsia de testículo (TESE: testicular sperm extraction): $32(6,1 \%)$; emprego de sêmen de doador (indivíduos azoospérmicos dos quais se tentou extrair espermatozóides do epidídimo ou testículos sem sucesso) duas $(0,4 \%)$; descongelamento de embriões em que foi usado sêmen doado: uma $(0,2 \%)$; descongelamento de embriões em que foram usados espermatozóides de PESA/TESE: três $(0,6 \%)$; e doação de óvulos e PESA/TESE: uma (0,2\%). Quanto aos outros $67,9 \%(353 / 520)$ das crianças, nenhum procedimento adicional à ICSI foi realizado. A Tabela 2 separa as crianças nascidas pela ocorrência ou não de técnica adicional e por tipo de gestação (única ou múltipla). 
Tabela 2 - Separação das crianças segundo a técnica de ICSI (e procedimento adicional utilizado) e tipos de gestação.

\begin{tabular}{|c|c|c|c|c|}
\hline Técnica + variantes $n=520$ & Únicos $n=250$ & Gêmeos n = 94 & Trigêmeos $n=60$ & Quadrigêmeos $n=16$ \\
\hline ICSI $n=353(67,9 \%)$ & 169 & 130 & 42 & 12 \\
\hline ICSI+Descongelamento de embrião $n=94(18,1 \%)$ & 51 & 34 & 9 & \\
\hline ICSI+Doação de óvulos $n=34(6,5 \%)$ & 17 & 8 & 9 & \\
\hline ICSI+PESA/TESE $n=32(6,1 \%)$ & 8 & 20 & & 4 \\
\hline ICSI+Doação de sêmen $n=2(0,4 \%)$ & & 2 & & \\
\hline ICSI+PESA/TESE+Descongelamento de embrião $n=3(0,6 \%)$ & 3 & & & \\
\hline ICSI+Doação de sêmen+Descongelamento de embrião $n=1(0,2 \%)$ & 1 & & & \\
\hline ICSI+Doação de óvulos+PESA/TESE $n=1(0,2 \%)$ & 1 & & & \\
\hline
\end{tabular}

ICSI: intracytoplasmic sperm injection/injeção intracitoplasmática de espermatozóides; PESA: percutaneous epididymal sperm aspiration; TESE: testicular sperm extraction.

Para a análise das malformações congênitas utilizamos a $10^{a}$ revisão da classificação internacional de doenças (CID-10). Nesse estudo analisamos apenas as MCM, isto é, malformações que causam prejuizo da função do órgão ou exigem correção cirúrgica, por serem as que têm relevância clínica. Além disso, como nem todas as crianças foram examinadas, não foi possivel fazer controle de malformações congênitas menores (isto é, que não provocam prejuízo da função e não requerem correção cirúrgica) porque facilmente passam desapercebidas pelos pais e são motivos de controvérsia até entre profissionais treinados.

Como a CID-10 não classifica as malformações em maiores ou menores, para essa divisão empregamos a classificação publicada por Bonduelle et al. ${ }^{12}$. Esses autores, que aceitam a definição mais difundida de malformações maiores e menores (descrita acima), publicaram lista com 237 itens de malformações consideradas menores, baseados no livro-texto de Aase. Essa reclassificação tem conotação prática clínica, dividindo as malformações por órgãos e regiões e levando em conta a gravidade da alteração (se causa prejuízo à função ou não).

A incidência de MCM em crianças nascidas vivas nesse estudo foi comparada com a obtida na população geral pelo Estudo Colaborativo Latino-Americano de Malformações Congênitas ${ }^{19}$ (ECLAMC), um registro de malformações congênitas que avalia as crianças nascidas em hospitais de referência. $\mathrm{Na}$ análise, utilizamos os resultados gerais do ECLAMC (RAE documento final XXXIV 2002) considerando todos os países participantes e os resultados somente com os dados referentes aos hospitais brasileiros.

Os dados foram analisados estatisticamente do programa InStat 3.0 para MacIntosh (GraphPad Software, San Diego, CA, EUA), empregando nos cálculos o teste do $\chi^{2}$. O nivel de significância foi determinado em $\mathrm{p}<0.05$.

\section{Resultados}

Foram identificados 15 casos de malformações consideradas maiores nas 520 crianças avaliadas no estudo, dando uma incidência de 2,9\%. Das 15 MCM encontradas, seis foram observadas em crianças submetidas ao questionário (40\%) e nove entre as crianças examinadas $(60 \%)$.

O ECLAMC obteve taxa de MCM em nascidos vivos de $2,1 \%$ (74.604/3.512.234). Ao separarmos os hospitais brasileiros que reportam ao ECLAMC e analisarmos a mesma taxa de MCM em nascidos vivos, observamos incidência de 2,6\% (22.115/ 853.227). Ao comparar o nosso resultado com o do ECLAMC geral e com o do ECLAMC-Brasil, não houve diferença significativa $(0,9$ e 0,7 ,respectivamente).

Quanto ao tipo de MCM, foram encontradas três crianças com malformações renais (dois casos de estenose de junção pielocalicial e um caso de hidronefrose unilateral), duas com defeito de fechamento do tubo neural (um caso de mielomeningocele associada à hidrocefalia e um caso de anencefalia), duas com defeito crânio-facial (lábio leporino e craniossinostose), quatro com cardiopatias isoladas (comunicação interventricular (CIV), comunicação interatrial (CIA), persistência do canal arterial (PCA) e estenose de artéria pulmonar), uma com malformação genital (criptorquidia bilateral que necessitou de correção cirúrgica), duas com sindrome de Down associadas a cardiopatia (CIV e PCA em conjunto com CIA) e uma com luxação congênita do quadril.

Em 10 dos 15 casos de MCM, apenas a ICSI foi empregada $(66,6 \%)$. No restante, em três casos, além da ICSI, houve transferência de embriões congelados (20\%), em um doação de óvulo $(6,7 \%)$ e em um utilização de espermatozóides obtidos por TESE $(6,7 \%)$.

Seis MCM ocorreram em gestações únicas e nove em múltiplas: oito gemelares e uma trigemelar. A análise estatística não mostrou diferença na 
incidência de MCM entre as gestações únicas e múltiplas $(\mathrm{p}>0,05)$. A Tabela 3 discrimina cada tipo de MCM, correlacionando com a ICSI e suas variantes e o tipo de gestação (única ou múltipla).

Tabela 3 - Distribuição das malformações congênitas maiores encontradas segundo a região/órgão, procedimento adicional e tipo de gestação.

\begin{tabular}{|c|c|c|c|}
\hline Malformação congênita maior & CID10 & ICSI + procedimento adicional & Gestação \\
\hline Renal: estenose da junção pielo-ureteral & Q62.1 & ICSI + Descongelamento & Única \\
\hline Renal: estenose da junção pielo-ureteral & Q62.1 & ICSI + Doação de óvulo & Única \\
\hline Defeito do tubo neural: mielomeningocele + hidrocefalia & Q05.7 & ICSI & Trigemelar \\
\hline Defeito do tubo neural: anencefalia & Q00.0 & ICSI & Gemelar \\
\hline Face: lábio leporino & Q36.1 & ICSI + TESE & Gemelar \\
\hline Cardíaca: estenose da artéria pulmonar & Q25.6 & ICSI & Gemelar \\
\hline Cardíaca: defeito do septo atrial & Q21.1 & ICSI & Gemelar \\
\hline Cardíaca - PCA & Q21.4 & ICSI & Única \\
\hline Cardíaca - CIV & Q21.0 & ICSI + Descongelamento & Gemelar \\
\hline Síndrome de Down + cardiopatia - PCA + CIA & $\begin{array}{l}\text { Q90.9 } \\
\text { Q21.4 } \\
\text { Q21.1 }\end{array}$ & ICSI & Gemelar \\
\hline Membros inferiores: luxação congênitado quadril & Q65.4 & ICSI & Única \\
\hline
\end{tabular}

CIV: comunicação interventricular, CIA: comunicação interatrial, PCA: persistência do canal arterial; ICSI: intracytoplasmic sperm injection/injeção intracitoplasmática de espermatozóides; TESE: testicular sperm extraction.

\section{Discussão}

Desde o início da aplicação clínica das TRA, tem havido discussões sobre o impacto no curso das gestações e, em especial, sobre a saúde das crianças nascidas após esses procedimentos. A exposição às condições laboratoriais, a seleção não natural dos gametas, a técnica empregada, além de fatores de base tais como idade materna avançada e doenças reprodutivas subjacentes ou a coexistência de condições clínicas nos pais, podem produzir efeito negativo nos resultados das TRA. Nesse aspecto, a incidência de malformações congênitas associadas às TRA é fonte importante de preocupação.

Devido às suas características, a ICSI tem, ao menos teoricamente, um maior potencial teratogênico que a FIV 5,6,9,10. Podemos relacionar como riscos teóricos da ICSI: utilização de espermatozóides que possuem o potencial de transportar anormalidades genéticas (p.ex.: mutações da doença fibrocística, deleções do cromossomo Y); uso de espermatozóides com defeitos estruturais (p.ex.: falta de proteínas espermáticas, formas graves de teratozoospermia); danos químicos ou mecânicos

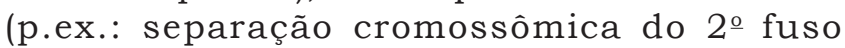

meiótico, ativação mecânica do oócito); introdução de materiais estranhos no oócito (contaminantes do meio de cultura, injeção de DNA estranho associado ao esperma). Por isso, os efeitos da ICSI sobre a prole concebida vêm sendo objeto de diversos trabalhos ${ }^{2-7,12,17}$.

Em estudos populacionais sobre malformações, a perda de seguimento de significativa proporção de indivíduos de amostra é sempre um problema importante, sendo inválido inferir que os casos perdidos sejam comparáveis àqueles incluídos na análise, em especial quando é esperado que a anormalidade em acompanhamento tenha incidência muito baixa ${ }^{9}$. Entretanto, essa é questão bastante presente. Nesse estudo tivemos perda de seguimento de $23,5 \%$ das crianças (160/680). Tal fato poderia ser explicado em parte pelo elevado número de crianças que são provenientes de diferentes pontos do Brasil, distantes do CRH-NADI. Além disso, alguns casais não mostraram interesse em participar da investigação, não trazendo seus filhos para serem avaliados nem fornecendo os dados sobre eles. Revisando a literatura, também observamos o problema da perda de seguimento, podendo vários fatores (formas de controle $\mathrm{e}$ recrutamento populacional, tamanho da amostra considerada, características locais de cada insti- 
tuição, tempo decorrido entre a avaliação das crianças e a realização das TRA/nascimento das crianças) influenciar a variação nos índices. Estudo prospectivo acompanhando gestações obtidas com ICSI refere perda de seguimento de $5,4 \%$ por falha no contato ou recusa das pacientes em permanecer no estudo ${ }^{16}$. Outro trabalho, acompanhando prospectivamente crianças de ICSI e FIV desde o nascimento, teve taxa de perda de seguimento aos dois meses de $18,8 \%$ para as crianças de ICSI e de $22,6 \%$ para crianças de FIV, mesmo após várias tentativas de contato ${ }^{12}$. Estudo multicêntrico em diferentes países avaliando crianças de ICSI aos cinco anos relatou perda de seguimento de metade $(307 / 607)$ por recusa dos pais ou falha no contato ${ }^{17}$. Outro estudo multicêntrico e multinacional, também avaliando crianças de ICSI aos cinco anos, informam indices diferentes para cada país entre as crianças contatadas e as que efetivamente participaram da avaliação, variando de um máximo de perda de $75 \%$ (Grécia) a um mínimo de 4\% (Suécia) ${ }^{2}$.

Geralmente é aceitável incidência de 3\% de MCM para a população geral ${ }^{5-9}$. Nesse estudo, foram identificados 15 casos de MCM nas 520 crianças nascidas vivas observadas. Isso dá incidência de MCM da ordem de 2,9\%, o que não foi diferente em comparação aos dados do ECLAMC geral e brasileiro. É importante lembrar que as crianças do ECLAMC foram avaliadas apenas no seu nascimento e, ao contrário, as crianças do nosso estudo foram analisadas entre um e cinco anos. Como algumas malformações manifestam-se ou são notadas pelos pais e/ou médicos mais tardiamente e a evolução do quadro pode modificar a classificação da malformação entre maior ou menor (p.ex.: necessidade de correção cirúrgica, fechamento espontâneo de defeito de septo cardíaco), essa diferença no momento da avaliação das crianças entre as populações de estudo e controle pode ter se refletido nas taxas de $\mathrm{MCM}^{2,4-17}$.

Devemos ressaltar também que a média de idade das mães à época do parto nesse estudo (34 $\pm 4,9$ anos) é mais elevada do que a das mães que concebem naturalmente, que é de $24,8^{20}$ ou entre 27 e 28 anos $^{9}$, fato observado na maioria dos trabalhos ${ }^{2,16}$. Isso poderia em parte explicar as malformações observadas decorrentes de aneuploidias. O risco de anormalidade cromossômica cresce com o aumento na idade da mulher, sendo a sindrome de Down a mais freqüente. Duas crianças com sindrome de Down foram encontradas nessa avaliação, em pacientes com idade ao parto maior que a média de idade da população do estudo (38 e 42 anos). Porém, para uma análise melhor sobre risco da síndrome de Down é importan- te aumentar a amostra populacional avaliada, considerando também que não é freqüente o seu registro em niveis aumentados ${ }^{3-7}$.

Por outro lado, alguns autores acreditam que a população de casais inférteis é de maior risco para transmitir alterações genéticas. Em um estudo foi examinado o cariótipo de 2650 casais (5300 pacientes) que foram submetidos à ICSI. Foram encontrados 162 cariótipos anormais $(3,1 \%)$, mais freqüente nos homens inférteis ${ }^{21}$. Outro estudo avaliou o cariótipo de 2196 homens e $1012 \mathrm{mu}$ lheres que seriam submetidos à ICSI devido a fator masculino de infertilidade durante período de 3 anos. Foi encontrada taxa de $6,1 \%$ de carótipos anormais nos homens, bem acima dos 3\% encontrados na população masculina fértil que servia de controle ${ }^{14}$. Surpreendentemente, alta porcentagem de anormalidades genéticas também foi relatada entre as mulheres (4,8\%). Além disso, outros autores observaram diminuição no risco de MCM em crianças concebidas por ICSI quando há correção para variáveis como idade materna, nível educacional, classe socioeconômica, fumo, bebida e gestações prévias, sugerindo que elas poderiam estar associadas também a características parentais e não apenas à técnica de $\mathrm{ICSI}^{2}$. Fazendo correção semelhante, outro estudo observa redução no risco de $\mathrm{MCM}^{16}$. Essas observações confirmam a idéia de que os casais inférteis são uma população especial de pacientes.

O resultado desta avaliação foi semelhante ao de outros trabalhos que não observaram risco aumentado de $\mathrm{MCM}$ em crianças concebidas por ICSI. Os resultados de amplo estudo comparativo mostraram que anomalias congênitas não são mais freqüentes em crianças nascidas após FIV ou ICSI do que nas crianças naturalmente concebidas $^{12}$. Nesse estudo a taxa de MCM para os nascidos vivos após ICSI foi de 3,4\%, para perdas baixas de seguimento ao nascimento $(1,5 \%)$ e de abortamento $(13,9 \%)$, o que reforça o resultado. Em outro estudo analisando 730 crianças, foram encontraram taxas de MCM de 2,2\% em nascidos vivos e de $2,7 \%$ para o total da população estuda$\mathrm{da}^{11}$, com taxa de perda de seguimento de $8,1 \%$ e de abortamento de 9,8\%.

Revisão da literatura envolvendo 11 trabalhos sobre o resultado das gestações e nascimentos após ICSI não encontrou aumento na incidência de MCM. Conclui ao final que a ICSI é técnica segura para ser aplicada aos casais inférteis, apesar de chamar a atenção sobre os possiveis vieses deste tipo de estudo (classificações das malformações diversas, avaliação das crianças em faixas etárias diferentes, grupos controle $)^{6}$.

Contudo, trabalhos mais recentes divergem 
desse baixo risco, mostrando aumento na incidência de malformações nas crianças nascidas após a aplicação da ICSI. Extenso estudo prospectivo analisando os nativivos, natimortos e os abortamentos acima de 16 semanas de gravidez observou taxa geral de MCM de $8,7 \%$ para ICSI e $6,1 \%$ para a população geral ${ }^{16}$. O fato de as populações de estudo e controle terem proporções similares de nascidos vivos, nascidos mortos e de abortamentos reforça a importância dos achados. Entretanto, nesse trabalho, o grupo controle restringese a uma região fixa na Alemanha, sendo os casos de ICSI de diferentes partes do país. Este é um dado que coloca viés sobre o grupo controle e que deve ser levado em consideração na interpretação dos resultados. Por outro lado, a classificação das MCM seguiu o sistema do European Registry of Congenital Anomalies and Twins e não o CID-10.

Estudo realizado em três centros de diferentes países (Bélgica, Suécia e Estados Unidos), avaliando crianças aos cinco anos de idade, encontrou $6,3 \%$ de $\mathrm{MCM}$, taxa significativamente superior aos 3\% encontrado no grupo controle composto por crianças naturalmente concebidas da população geral ${ }^{17}$. Porém, neste estudo apenas em um centro (Bélgica) a diferença na incidência de MCC foi significativa. Nos outros dois centros, a taxa de MCM foi muito silimilar à da população geral. Além disso, a disparidade nas taxas de MCM pode ter sido influenciada por viés no recrutamento das crianças. No centro belga o grupo controle foi recrutado nas escolas, o que pode ter subestimado a incidência de MCM já que crianças com anomalias graves podem não conseguir freqüentar escolas normais ou mesmo podem não ter sobrevivido até os cinco anos devido à intensidade do quadro. No centro americano, o recrutamento do grupo controle foi feito por anúncio, porém os pais das crianças com complicações médicas podem ter preferido não expor suas crianças a mais testes.

Em estudo multicêntrico envolvendo cinco países (Reino Unido, Bélgica, Dinamarca, Suécia e Grécia), também avaliando crianças nascidas de ICSI aos cinco anos, encontrou-se taxa de MCM de $4,2 \%^{2}$. Comparando com as crianças concebidas naturalmente na mesma faixa etária, o risco encontrado para MCM foi 2,7 maior para as crianças de ICSI. Contudo, após correção de algumas variáveis como idade materna, nível educacional, classe socioeconômica, fumo, bebida e gestações prévias, houve queda para 2,5 no risco de MCM, sugerindo que elas poderiam estar associadas também a características parentais e não apenas à técnica de ICSI. Por outro lado, como em grande parte dos centros (Reino Unido, Bélgica e Grécia) as crianças do grupo controle foram recrutadas nas escolas, os autores admitem possivel viés que poderia diminuir a taxa de malformação. Porém os autores também referem falhas no recrutamento das crianças de ICSI, devido à recusa dos pais em participar da avaliação.

Recentemente, revisão sistemática avaliou a prevalência de defeitos congênitos em crianças concebidas por ICSI e FIV em comparação a crianças concebidas naturalmente. De 25 estudos identificados, oito mostraram risco de $25 \%$ de malformações em FIV/ICSI, ao passo que 7 sugeriram $30-40 \%$ de risco ${ }^{4}$. Porém, além de esta revisão não fazer a separação das MCM, a análise das malformações foi em conjunto para FIV e ICSI. Do mesmo modo, meta-análise analisando 19 estudos sugere aumento do risco de MCM de 29\% para crianças concebidas por FIV ou ICSI, mas também não individualiza as técnicas ${ }^{3}$. Na verdade, os resultados dos vários estudos enfatizam a necessidade de monitoramento contínuo das crianças submetidas à ICSI.

Por outro lado, Bonduelle et al. ${ }^{2}$ relatam aumento das MCM nas crianças de ICSI devido à taxa mais elevada em meninos $(8,2 \%)$ comparada às meninas $(3,6 \%)$, principalmente pelo excesso de malformações urogenitais. Esses autores observaram 3,7\% (20 casos) de malformações urogenitais na ICSI, com predomínio de hipospádias, incidência estatisticamente maior que na FIV $(2,1 \%)$ e no controle $(1 \%)$. Outros autores reafirmam o risco de malformações urogenitais, particularmente hipospádia, em crianças de ICSI $^{6-8}$. Porém, nossos dados não confirmam essa maior incidência. Das 15 malformações encontradas no estudo, quatro foram urogenitais, correspondendo a 26,7\% (4/15) das MCM encontradas e, mais importante, nenhum caso de hipospádia foi observado. Como nossa amostra populacional de ICSI (520 crianças) é muito similar à dos outros estudos, provavelmente variáveis como diferenças demográficas podem influenciar a incidência desse tipo de malformação. Alguns trabalhos também não observaram aumento significativo na incidência de hipospádia em crianças concebidas por $\mathrm{ICSI}^{7,16}$.

As MCM mais encontradas foram as de origem cardiaca, que corresponderam a 40\% total (6/ 15). Tivemos quatro casos de malformações cardíacas isoladas e dois casos de malformações cardíacas associadas: um caso de CIV com sindrome de Down e um de PCA e CIA com síndrome de Down. Outros autores também estão de acordo com esses achados. Em um estudo foram encontrados cinco casos de malformação cardiaca em crianças de ICSI entre $16 \mathrm{MCM}(31,2 \%)^{11}$. Outra avaliação encontrou 30 casos de malformações cardía- 
cas em de um total de 95 MCM $(31,6 \%)^{12}$. A análise de 3.372 crianças mostrou aumento de 1,5\% no risco de malformação cardíaca em crianças de ICSI em relação a população geral ${ }^{16}$. Por outro lado, Koivurova et al. ${ }^{1}$ mostram risco maior de malformação cardíaca também em crianças nascidas após FIV (40\%, oito casos/20 malformações) em comparação à população geral (20\%, cinco casos / 25 malformações). Recente revisão da literatura não mostrou diferença nas taxas de malformação cardiaca entre crianças provenientes de ICSI em comparação às de $\mathrm{FIV}^{7}$. Assim, as anormalidades cardíacas provavelmente não estão diretamente relacionadas à técnica da ICSI.

Encontramos um caso de mielomeningocele com hidrocefalia e um caso de anencefalia, que representaram 13,3\% (2/15) do total das MCM e $0,4 \%(2 / 520)$ do total de crianças. A literatura tem mostrado também baixa incidência de malformações do sistema nervoso. Estudo prospectivo encontrou 21 crianças de ICSI de um total de 3.372 com malformação do sistema nervoso central $(0,6 \%)$, incidência igual à da população geral que servia de controle, que apresentou 48 crianças comprometidas em amostra de $8.016(0,6 \%)^{16}$. Contudo, há relato de aumento no risco de malformações do sistema nervoso central com aplicação de TRA $^{8}$.

Das $15 \mathrm{MCM}$ encontradas, nove ocorreram em gestações múltiplas. Tal fato poderia apontar para uma associação entre essas duas situações. A incidência de MCM nas gestações únicas foi de $2,4 \%$ e nas múltiplas de $3,3 \%$ (4,1\% nas gemelares e 1,6\% nas trigemelares), porém a análise estatística não mostrou diferença significativa ( $>0.05)$. Entretanto muitos trabalhos fazem correlação positiva entre a prematuridade decorrente das gestações múltiplas e maior taxa de $\mathrm{MCM}^{3,5,16,22}$.

Apenas um caso de malformação após ICSI estava associado ao emprego de TESE, número insuficiente para tecer qualquer comentário. Contudo, na literatura, quando crianças nascidas por ICSI foram separadas com relação à origem dos espermatozóides (testicular e epididimário) e comparadas com grupo controle em que os espermatozóides foram obtidos do ejaculado, não se observou aumento na incidência de malformações congênitas ${ }^{5}$.

Tivemos três casos de MCM que ocorreram em embriões de ICSI descongelados $(20 \%$ do total), sendo dois desses casos em gestações gemelares. A literatura não relata dados negativos quanto à segurança das técnicas de criopreservação, e até o momento os estudos de acompanhamento de crianças nascidas após descongela- mento não demonstram ligação entre a técnica e a incidência de malformações ${ }^{3,4,6}$.

Alguns aspectos importantes precisam ser considerados quando estudos populacionais sobre malformações congênitas são feitos ${ }^{9,23}$ : o grupo controle empregado, diferenças nas avaliações entre o grupo controle e de estudo, as taxas de desistência do estudo, análise individualizada de cada tipo de TRA, o tamanho da amostra populacional, o tempo de seguimento e a realização ou não de exame físico. Porém, alguns deles são extremamente dificeis de serem controlados, produzindo vieses importantes nesses estudos.

Além disso, todos acreditam na necessidade de estudos multicêntricos, colaborativos, que espelhem com maior fidedignidade as variações populacionais e que acompanhem prospectivamente as crianças por um longo periodo de tempo (cinco anos), com grupo controle populacional casado desde o início, com controle das variáveis mais importantes, e com amostra populacional grande o suficiente. Essa seria a forma ideal para avaliar a real incidência de malformações congênitas maiores no mundo, em especial após a ICSI.

Em conclusão, as crianças concebidas por ICSI e nascidas vivas apresentam incidência de MCM (2,9\%), próxima ao esperado para a população geral $(2,6 \%)$. Entretanto, para estabelecer com precisão os riscos de MCM é necessária continuidade na avaliação das crianças concebidas por ICSI.

Que tenhamos conhecimento, esse é o primeiro relato no Brasil sobre a incidência de malformações em crianças concebidas por ICSI. Apesar de todos os vieses inerentes a esse tipo de pesquisa, muitas vezes de dificil solução, os dados desse trabalho podem servir de base para a organização de programa de seguimento dessas crianças organizado em âmbito nacional e latino-americano, para que tenhamos resultados adequados à nossa realidade dos nascimentos de ICSI e de outras técnicas de reprodução assistida no Brasil e na América Latina.

\section{Referências}

1. Koivurova S, Hartikainen AL, Gissler M, Hemminki E, Sovio U, Järvelin MR. Neonatal outcome and congenital malformations in children born after invitro fertilization. Hum Reprod. 2002;17(5):1391-8.

2. Bonduelle M, Wennerholm UB, Loft A, Tarlatzis BC, Peters C, Henriet S, et al. A multi-centre cohort study of the physical health of 5-year-old children conceived after intracytoplasmic sperm injection, 
in vitro fertilization and natural conception. Hum Reprod. 2005;20(2):413-9.

3. Rimm AA, Katayama AC, Diaz M, Katayama KP. A meta-analysis of controlled studies comparing major malformation rates in IVF and ICSI infants with naturally conceived children. J Assist Reprod Genet. 2004;21(12):437-43.

4. Hansen M, Bower C, Milne E, de Klerk N, Kurinczuk JJ. Assisted reproductive technologies and the risk of birth defects - a systematic review. Hum Reprod. 2005;20(2):328-38.

5. Aboulghar MA. Perinatal complications of assisted reproduction. Croat Med J. 2005;46(5):751-8.

6. Retzloff MG, Hornstein MD. Is intracytoplasmic sperm injection safe? Fertil Steril. 2003;80(4):851-9.

7. Lie RT, Lyngstadaas A, Orstavik KH, Bakketeig LS, Jacobsen G, Tanbo T. Birth defects in children conceived by ICSI compared with children conceived by other IVF-methods; a meta-analysis. Int $\mathrm{J}$ Epidemiol. 2005;34(3):696-701.

8. Källen B, Finnström O, Nygren KG, Olausson PO. In vitro fertilization (IVF) in Sweden: risk for congenital malformations after different IVF methods. Birth Defects Res A Clin Mol Teratol. 2005;73(3):162-9.

9. Van Steirteghem AC, Bonduelle M, Devroey P, Liebaers I. Follow-up of children born after ICSI. Hum Reprod Update. 2002;8(2):111-6.

10. Chang AS, Moley KH, Wangler M, Feinberg AP, Debaun MR. Association between BeckwithWiedemann syndrome and assisted reproduction technology: a case series of 19 patients. Fert Steril. 2005;83(2):349-54.

11.Loft A, Petersen K, Erb K, Mikkelsen AL, Grinsted $\mathrm{J}$, Hald F, et al. A Danish national cohort of 730 infants born after intracytoplasmic sperm injection (ICSI) 1994-1997. Hum Reprod. 1999;14(8):2143-8.

12. Bonduelle M, Liebaers I, Deketelaere V, Derde MP, Camus M, Devroey P, et al. Neonatal data on a cohort of 2889 infants born after ICSI (1991-1999) and of 2995 infants born after IVF (1983-1999). Hum Reprod. 2002;17(3):671-94.

13. Jozwiak EA, Ulug U, Mesut A, Erden HF, Bahceci M. Prenatal karyotypes of fetuses conceived by intracytoplasmic sperm injection. Fertil Steril. 2004;82(3):628-33.

14. Gekas J, Thepot F, Turleou C, Sittroi JP, Dadoune JP, Briault S, et al. Chromosomal factors of infertility in candidate couples for ICSI: an equal risk of constitutional aberrations in women and men. Hum Reprod. 2001;16(1):82-90.

15.Jacobs PA, Browne C, Gregson N, Joyce C, White $\mathrm{H}$. Estimates of the frequency of chromosome abnormalities detectable in unselected newborns using moderate levels of banding. J Med Genet. 1992;29(2):103-8.

16. Katalinic A, Rösch C, Ludwig M; German ICSI FollowUp Study Group. Pregnancy course and outcome after intracytoplasmic sperm injection: a controlled, prospective cohort study. Fertil Steril. 2004;81(6):1604-16.

17.Bonduelle $\mathrm{M}$, Bergh $\mathrm{C}$, Niklasson A, Palermo GD, Wennerholm UB; Collaborative Study Group of Brussels, Gothelburg and New York. Medical followup study of 5-year-old children. Reprod Biomed Online. 2004;9(1):91-101.

18. REDLARA. Red Latinoamericana de Reproducción Asistida [homepage de la Internet]. Registro Latinoamericano (RLA). Santiago; 2002. [citado 2005 Ago 9]. Disponible en: http://www.redlara.com/reg_2002.asp.

19. ECLAMC. Documento final de la $34^{\mathrm{a}}$ Reunion Anual del Estudio Colaborativo Latinoamaricano de Malfomaciones Congênitas [conference on the Internet]; 14-19 ago. 2002; Mangaratiba, Brasil. Rio de Janeiro: FIOCRUZ; 2002 [citado 2005 Jan 4]. Disponible em: http://www.eclamcnet.net

20.IBGE. Instituto Brasileiro de Geografia e Estatística. Diretoria de Pesquisas (DPE). Coordenação de População e Indicadores Sociais (COPIS) [homepage da Internet]. Perfil socioeconômico da maternidade nos extremos do período reprodutivo (Primeira Versão). Maio 2005 [citado 2005 Set 16]. Disponivel em: http://www.ibge.gov.br/home/estatistica/ populacao/perfil_maes/Maes_jovens_e_maduras.pdf.

21. Kayed HF, Mansour RT, Aboulghar MA, Serour GI, Amer AE, Abdrazik A. Screening for chromosomal abnormalities in 2650 infertile couples undergoing ICSI. RBM Online [serial on the Internet]. 2006 Mar [cited 2006 Jan 20];12(3). Available from: http:// www.rbmonline.com/Article/2034.

22. Kuwata T, Matsubara S, Ohkuchi A, Watanabe T, Izumi A, Honma Y, et al. The risk of birth defects in dichorionic twins conceived by assisted reproduction technology. Twins Res. 2004;7(3):223-7.

23. Ludwig M. Development of children born after IVF and ICSI. Reprod Biomed Online. 2004;9(1):10-2. 